\title{
DURABILIDAD DE LAS ARMADURAS EN EL SISTEMA DRAGADOS-PLASTBAU
}

\author{
M.a Carmen Andrade Perdrix, IETcc/CSIC \\ Jesús Rodriguez Santiago, Dragados y Construcciones, S. A.
}

\section{CONSIDERACIONES PREVIAS}

En un sistema de las caracteristicas del presente, los elementos metálicos que pueden sufrir corrosión son principalmente la malla y los conectores que atraviesan el poliestireno. La porosidad del microhormigón y el no siempre suficiente espesor de recubrimiento pueden permitir una carbonatación del microhormigón que alcance la malla durante el periodo de vida útil previsto para la estructura. Este riesgo de corrosión por carbonatación viene acrecentado en este sistema particular por una posible aparición de fisuras (retracción, movimientos de dilatación y asentamientos diferenciales, etc.).

Por otro lado, la existencia de conectores metálicos que atraviesen el poliestireno para unir las mallas entre si, introduce un motivo de incertidumbre sobre el comportamiento de estos elementos. Si bien el poliestireno es inerte en cuanto a su interacción con el metal, las perforaciones que producen los conectores al atravesarlo, pueden almacenar restos del agua de amasado que podrian tener alcalinidad inferior (por dilución) a la de la propia fase acuosa del microhormigón, pudiendo desencadenar algún fenómeno de corrosión.

La corrosión provocada por la presencia de cloruros sólo se debe considerar en el caso de que las construcciones con este sistema se efectúen en ambientes marinos. Se optó pues por no realizar ensayos que tuvieran en cuenta la presencia de cloruros, ya que por un lado la adición de los mismos al agua de amasado se aleja de la situación real de una estructura situada en dichos ambientes y por otro lado, someter las probetas a la acción de disoluciones de cloruros da lugar a ensayos de muy larga duración, debido a que éstos difunden lentamente. Además se conoce por experiencias anteriores del grupo del IETcc, que trabaja en corrosión de armaduras, que el acero inoxidable soporta sin corroerse, cantida- des muy elevadas de cloruros, y que el acero galvanizado puede también resistir cantidades superiores a las que despasivan el acero normal si el espesor y las caracteristicas del galvanizado son los adecuados. Asimismo se conoce que una relación de nitritos/cloruros $\geqslant 2 / 1$ bloquea la corrosión y mantiene pasivo el acero normal o galvanizado.

Asi pues, en los ensayos efectuados se estudió solamente la evolución de la corrosión de la malla y los conectores con el tiempo y en algunos casos se sometieron las probetas a carbonatación acelerada. No se contempló en el estudio el comportamiento de las armaduras embebidas en los pilares de los muros D-P II, ni en el forjado Cádiz, ya que éstas se consideran estructuras tradicionales.

En cuanto a la vida útil previsible, dado que el microhormigón de los muros de D-P II cumplen una misión de acabado, se consideró para ellos una vida útil mínima de 10 años. En cambio, como los muros D-P I cumplen una misión resistente, las prescripciones finales tienen como objeto garantizar una vida útil mínima de 50 años, al igual que en el caso de otras estructuras de edificación.

Debido a que este sistema constructivo está basado en unos espesores de recubrimiento de la malla limitados, para asegurar las vidas útiles previstas fue necesario contar en algunos casos con las pinturas o revestimientos de acabado de los muros y además estudiar otros métodos de protección adicionales. Los métodos considerados fueron:

a) mallas y conectores galvanizados,

b) conectores de acero inoxidable,

c) uso de un aditivo inhibidor de la corrosión de armaduras (nitrito cálcico) añadido al agua de amasado del microhormigón. 


\section{ENSAYOS EFECTUADOS}

\subsection{Tipos de ensayos y probetas}

Se ensayaron probetas de microhormigón y probetas sandwich de diferentes tamaños, así como paneles a escala real estudiándose la evolución de la corrosión de las armaduras en el tiempo cuando se sometieron a ciclos de humedad-sequedad y en algún caso a carbonatación acelerada del microhormigón en un momento dado.

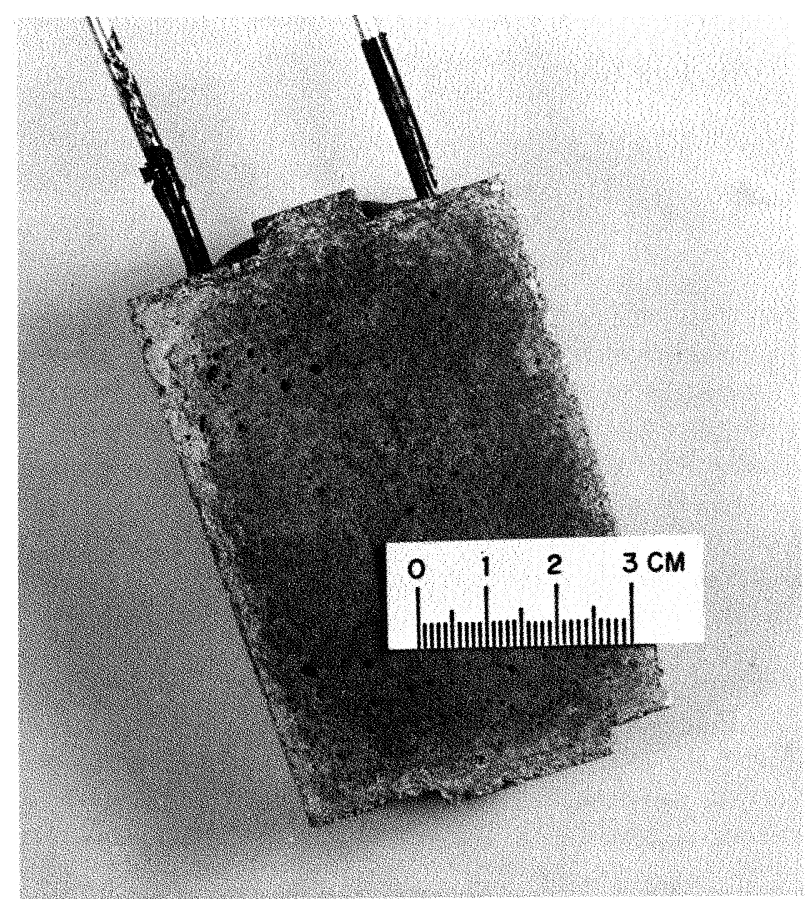

Fig. 1. - Probetas de microhormigón de $8 \times 5 \times 2 \mathrm{~cm}$

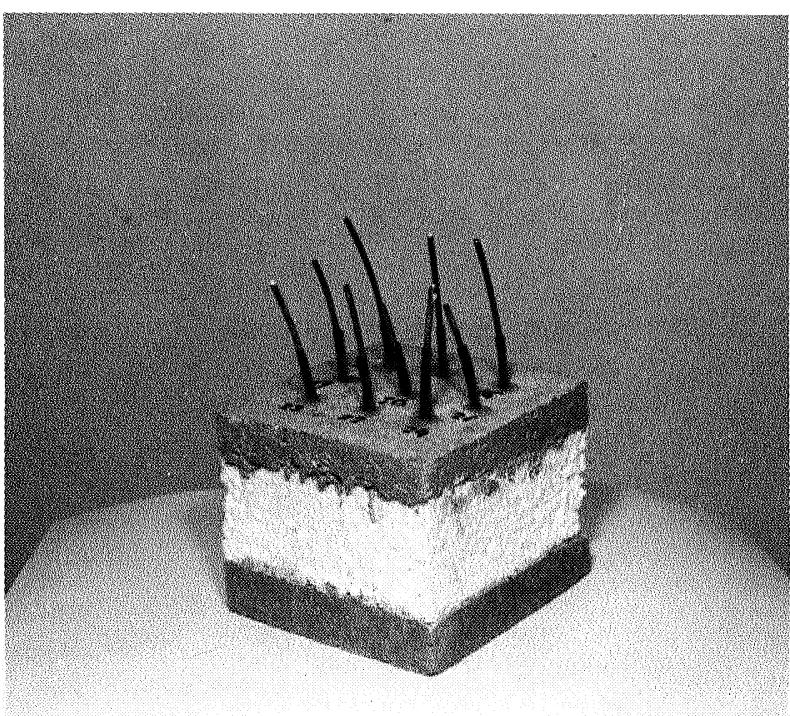

Fig. 2. - Probetas $20 \times 20 \times 12 \mathrm{~cm}$ para el ensayo de los conectores.

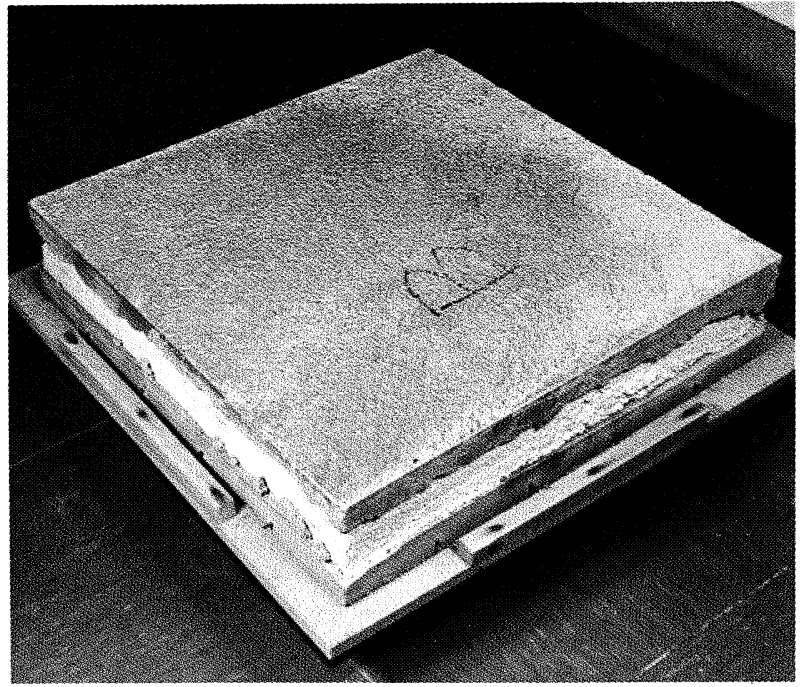

Fig. 3. - Probeta de $54 \times 54 \times 12 \mathrm{~cm}$ para el ensayo conjunto de mallas y conectores.

Se fabricaron las probetas para estudiar por separado el comportamiento del acero (negro, galvanizado o inoxidable) en el microhormigón, el de los conectores en el sistema microhormigón poliestireno - microhormigón y el del conjunto malla-conectores en las probetas sandwich. Las figuras 1,2 y 3 muestran tres tipos de probetas empleadas fabricándose en cada caso con y sin inhibidor $\left(3 \%\right.$ de $\left(\mathrm{NO}_{2}\right)_{2} \mathrm{Ca}$ en relación al peso de cemento). La velocidad de la corrosión del acero, tanto en malla como en conectores, se evaluó mediante técnicas electroquímicas, gavimétricas o de simple observación visual.

La incidencia de las presencias de fisuras en el microhormigón, asi como la influencia de un ambiente especialmente húmedo y cálido se evaluó estudiando el estado de la malla y los conectores embebidos en los paneles sometidos al choque térmico (fig. 4).

Se determinó también la porosidad del microhormigón en diferentes muestras extraidas de elementos de grandes dimensiones y de las probetas preparadas para los ensayos de corrosión.

\subsection{Técnicas de medida}

La carbonatación acelerada se empleó para estudiar el comportamiento del acero en un medio cuya alcalinidad ha disminuido. Se llevó a cabo introduciendo las probetas en recipientes estancos por el que se hizo circular $\mathrm{CO}_{2}$ gas. El flujo de $\mathrm{CO}_{2}$ se mantuvo con periodicidad regular para desplazar todo el aire del recipiente y asegurar una atmósfera del $100 \%$ de $\mathrm{CO}_{2}$. Esta operación se daba por finalizada cuando las probetas no aumentaban de peso. 
El seguimiento de la evolución de la velocidad de corrosión con el tiempo se determinó mediante la técnica electroquímica denominada «Resistencia de Polarización" (Rp). Consiste básicamente en aplicar una pequeña polarización al acero de la armadura y registrar el cambio de intensidad. Luego, mediante la fórmula de Stern y Geary se calcula la velocidad instantánea de corrosión

$$
\mathrm{I}_{\text {corr }}=\mathrm{B}_{1} \frac{\Delta \mathrm{I}}{\Delta \mathrm{E}}=\frac{\mathrm{B}}{\mathrm{R}_{\mathrm{p}}}
$$

donde $B$ es una constante que depende del tipo de metal y del medio agresivo.

Para efectuar las medidas se necesitan, además del acero de la armadura, un electrodo auxiliar y otro de referencia (fig. 5). Se emplearon un potenciostato, un generador de funciones, un osciloscopio y un ordenador para la determinación periódica de la resistencia de polarización y se obtuvieron gráficos de la variación de la "l corr" en el tiempo para cada caso estudiado.

En las probetas de pequeño tamaño se procedió a la limpieza del óxido del acero antes de fabricarlas y después de finalizado el ensayo para por diferencia de pesada poder conocer la pérdida real de metal producida por el proceso corrosivo.

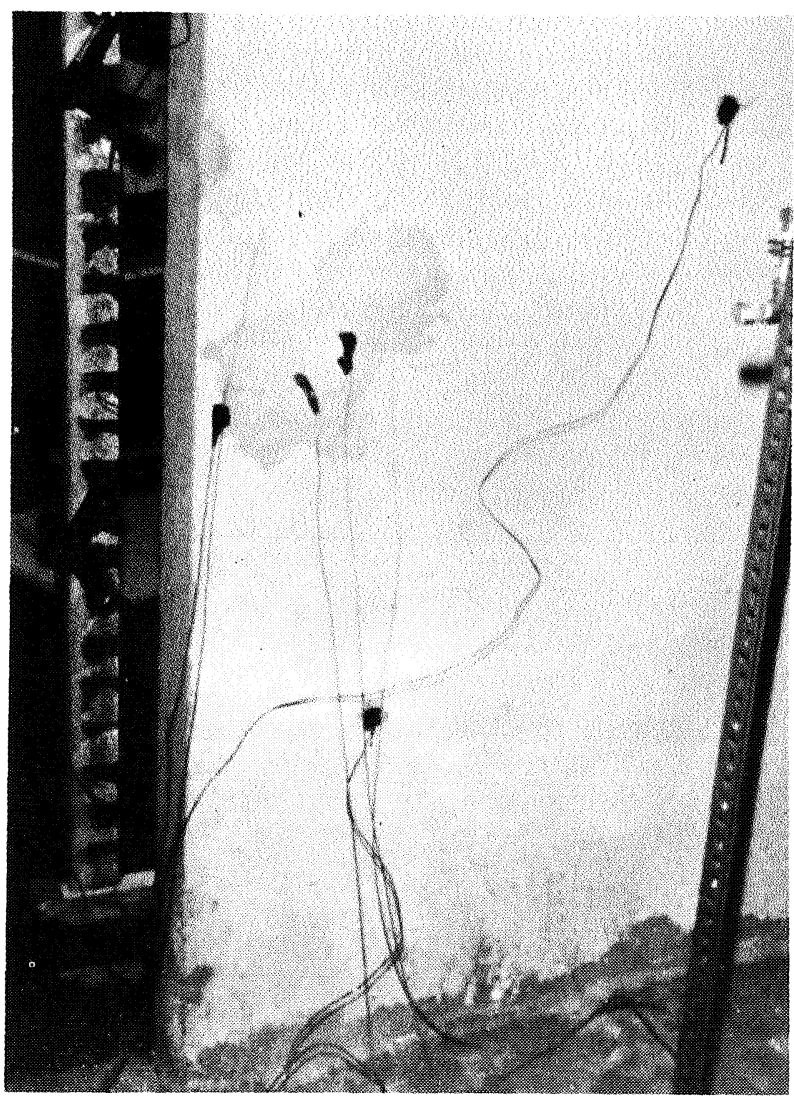

Fig. 4. - Panel del ensayo de choque termico.

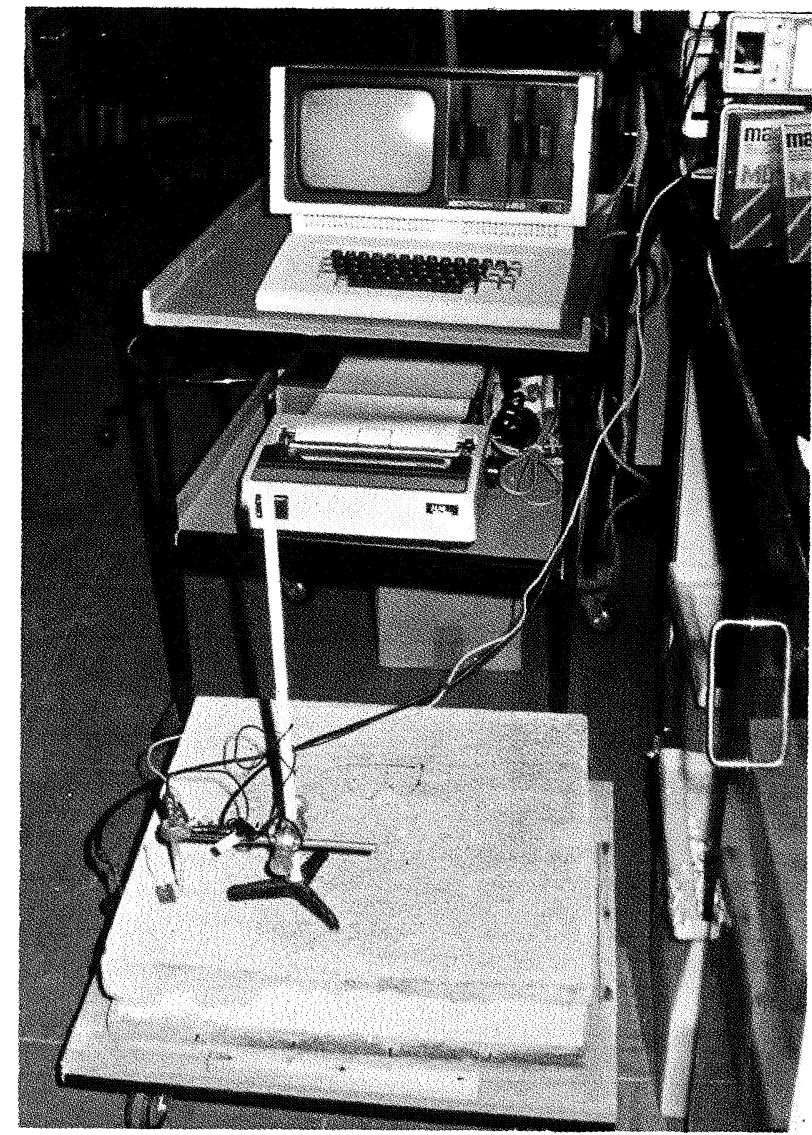

Fig. 5. - Instrumentación para la medida electroquimica de la velocidad de corrosión.

\section{RESULTADOS}

Las medidas de la porosidad del microhormigón proporcionaron valores ligeramente superiores al $20 \%$ (en volumen) en las probetas de los ensayos de corrosión, valores altos debidos al material y a la forma manual de fabricación de las probetas. Estos valores se situaron entre el 16 y $17 \%$ cuando se midió la porosidad de las muestras de los paneles preparados para otros ensayos en los que el microhormigón se colocó mediante proyección mecánica con los medios empleados habitualmente en la construcción de edificios con este sistema.

La medición de la profundidad del espesor de carbonatación en elementos conservados en laboratorio o a la intemperie proporcionó valores entre 1 y $6 \mathrm{~mm}$ para un periodo superior a un año. El pequeño número de resultados obtenidos y su erraticidad debido a las múltiples variables que intervienen en el proceso no permitieron obtener conclusiones para hacer predicciones sobre las profundidades de carbonatación probables en los 10 ó 50 años de vida útil de los elementos del sistema.

La medida de la velocidad de corrosión de las armaduras embebidas en probetas pequeñas de microhormigón puso de manifiesto el excelente 
comportamiento del acero inoxidable con el que se midieron velocidades de corrosión inferiores a la critica para diferentes condiciones de humedad e incluso después de la carbonatación del microhormigón (fig. 6).
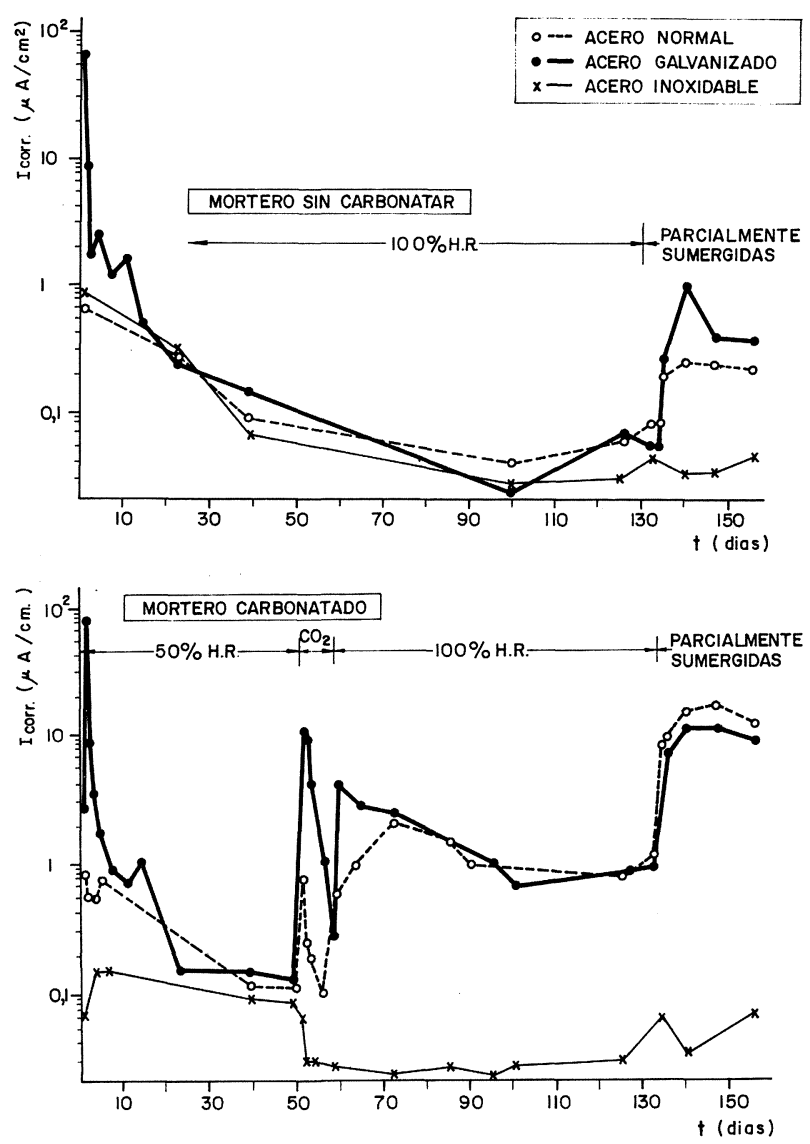

Fig. 6. - Relacion entre la intensidad de corrosion "corr" y el tiempo " $t$ " en armaduras embebidas en probetas de microhormigon de $8 \times 5.5$ $\times 2 \mathrm{~cm}$.

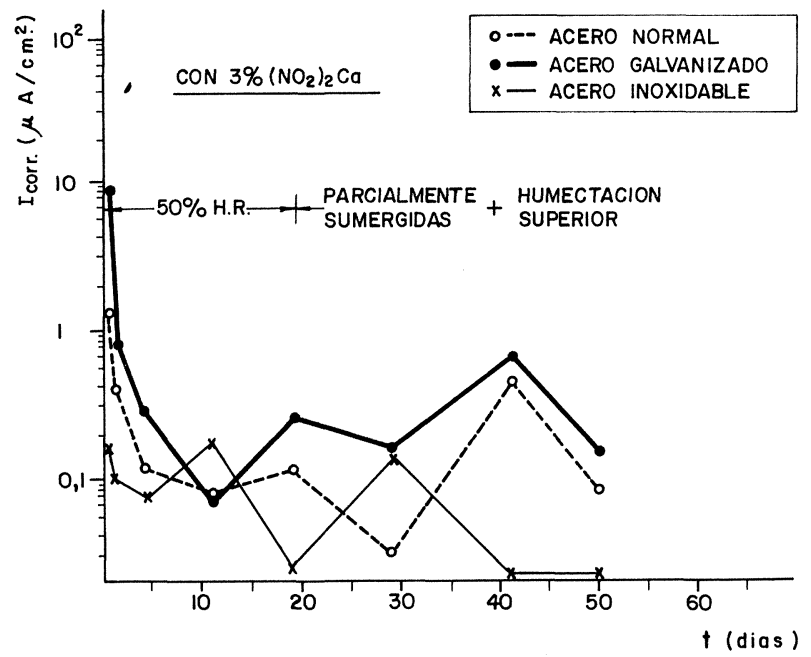

Fig. 7. - Relacion entre la intensidad de corrosion "lcorr" y el tiempo "t" en armaduras embebidas en probetas sandwich de $20 \times 20 \times 12 \mathrm{~cm}$.

Los redondos de acero sin proteger o galvanizados tuvieron un comportamiento similar entre si en las probetas anteriores, presentando valores de la velocidad de corrosión por encima de la critica en muestras carbonatadas y parcialmente sumergidas (fig. 6). Sin embargo, mientras la corrosión del galvanizado da lugar a óxidos que poco a poco lo pasivan, aun a costa de consumir parte de la capa galvanizada, la corrosión del acero sin protección produce una disminución de su sección resistente y la expansividad de los óxidos generados fisura o rompe la capa de microhormigón que lo rodea. La introducción en el agua de amasado de un $3 \%$ de $\left(\mathrm{NO}_{2}\right)_{2} \mathrm{Ca}$ en peso de cemento redujo la velocidad de corrosión del acero sin protección a valores diez veces menores de los medidos en la situación anterior.

Esta acción inhibidora del $\left(\mathrm{NO}_{2}\right)_{2} \mathrm{Ca}$ también se manifestó en el comportamiento de esos redondos en los ensayos con probetas con conectores (fig. 7). Por último los ensayos con las probetas sandwich arrojaron valores bajos de la velocidad de corrosión de las mallas y conectores si bien, dado el tamaño de la probeta, estos valores son promedio y no detectan corrosiones localizadas.

Terminados los ensayos las muestras fueron rotas y se extrajeron las mallas y redondos para su inspección visual y en su caso para proceder a su pesada. En las probetas pequeñas, sólo los redondos sin proteger con el microhormigón carbonatado aparecieron recubiertos de óxido pardo, lo que ya era de esperar a la vista de las medidas electroquimicas efectuadas. Tanto los redondos de las probetas con conectores como las armaduras de las probetas grandes manifestaron sólo en algunos de los casos ensayados una ligera corrosión superficial sin importancia.

Las armaduras de los paneles ensayados al choque térmico fueron también examinadas al terminar los ensayos. Los conectores, por los que en ocasiones circulaba el agua desde la cara ensayada a la posterior, se mostraron corroidos superficialmente aunque la disminución de sus diámetros y el óxido generado pueden considerarse despreciables en términos de vida útil del elemento, dadas las condiciones extremas del ensayo (fuertes oscilaciones de temperatura y humedad). Las mallas también aparecieron sólo ligeramente corroidas incluso en las zonas próximas a las fisuras producidas en el microhormigón durante el ensayo. Es de destacar que en el panel fabricado con malla galvanizada, ésta se mostró prácticamente intacta y sin ningún signo visible de corrosión al final del ensayo de choque térmico.

Las pérdidas gravimétricas medidas en los redondos de las probetas proporcionaron una información en consonancia con las medidas electroquimicas efectuadas.

\section{CONCLUSIONES Y RECOMENDACIONES DE USO}

Estos ensayos y la experiencia previa del equipo de corrosión del I.E.T. han conducido a las siguientes conclusiones: 
TABLA 1

Recomendaciones sobre el valor mínimo del espesor de recubrimiento de las armaduras en elementos del sistema Plastbau

\begin{tabular}{|c|c|c|c|c|}
\hline \multirow{2}{*}{ AMBIENTE } & \multirow{2}{*}{ I. } & \multicolumn{2}{|r|}{ II } & \multirow{2}{*}{ III } \\
\hline & & II a & II $b$ & \\
\hline$D-P I$ & $\begin{array}{l}15 \mathrm{~mm} . \\
10 \mathrm{~mm} .+ \text { PINTURA DE ACABADO * }\end{array}$ & $15 \mathrm{~mm}$. & PINTURA DE ACABADO* & $20 \mathrm{~mm}$. + PINTURA DE ACABADO*+GALVANIZADO ** \\
\hline$D-P$ II & $\begin{array}{l}10 \mathrm{~mm} . \\
5 \mathrm{~mm} .+ \text { CAPA DE TERMINACION }\end{array}$ & $10 \mathrm{~mm}$. & $\begin{array}{l}15 \mathrm{~mm} . \\
10 \mathrm{~mm} .+ \text { APLACADO EXTERIOR } \\
5 \mathrm{~mm}+\text { ALICATADO INTERIOR }\end{array}$ & $\begin{array}{l}20 \mathrm{~mm} . \\
15 \mathrm{~mm}++ \text { PINTURA DE ACABADO* } \\
15 \mathrm{~mm} .+ \text { GALVANIZADO** } \\
15 \mathrm{~mm}++ \text { NITRITOS ( } 3 \% \text { PESO CEMENTO) }\end{array}$ \\
\hline
\end{tabular}

* PINTURAS O REVESTIMIENTOS DE RESINAS SINTETICAS INSAPONIFICABLES DE LOS EMPLEADOS EN EXTERIORES CON ESPESORES MINIMOS DE PELICULA SECA DE 0,35 Y $1,00 \mathrm{~mm}$. PARA LAS PINTURAS Y REVESTIMIENTOS RESPECTIVAMENTE.

* * GALVANIZAdO de MALLA Y CONECTORES CON ESPESOR MINIMO $30-40 \mu \mathrm{m}$.

- El microhormigón ensayado se ha manifestado suficientemente protector de la armadura si bien su porosidad es elevada en comparación con un hormigón de mayor tamaño máximo de árido.

- La corrosión detectada en los conectores puede considerarse despreciable en términos de vida útil de la estructura pudiendo predecirse un fallo por corrosión de la malla antes que los conectores resulten dañados.

- De los métodos de protección ensayados el acero inoxidable presenta un óptimo comportamiento en todos los ambientes. El acero galvanizado y el empleo de $\left(\mathrm{NO}_{2}\right)_{2} \mathrm{Ca}$ alargan la vida útil del sistema.

Como resultado del trabajo llevado a cabo se establecieron unas recomendaciones de uso (tabla 1) en función del ambiente en el que se ubiquen los elementos constructivos, habiéndose definido los siguientes tipos de ambientes:

Ambiente I: Interiores secos (se excluyen cocinas, cuartos de baño y sótanos húmedos no ventilados). Ambiente lla: Exteriores secos. Ambiente Ilb: Interiores o exteriores con humedad frecuente elevada. Ambiente III: Interiores o exteriores en atmósferas agresivas (marinas o industriales).

No se recomendó el uso de conectores inoxidables ya que la dificultad que presentan para ser soldados y su precio elevado lo desaconsejaron y por otra parte porque se pudo contar con otros sistemas de protección adicional.

Actualmente se prosiguen ensayos complementarios para estudiar la capacidad protectora de diferentes tipos de pinturas o revestimientos del microhormigón frente a la acción de los cloruros o a la carbonatación con el fin de considerarlos como un método alternativo: de protección adicional (al empleo de galvanizado o nitritos) o de necesidad de aumento del espesor de recubrimientos.

\section{BIBLIOGRAFIA}

1. STERN, M. and GEARY, A. L.: «Electrochemical Polarization. I. A Theorical analysis of the shape of polarization curves". Journal of Electrochemical Soc. vol. 104, n.o 1 (1957), 56.

2. SATTERFIELD, D. T.; TONINI, D. E.: "The status of laboratory and field studies involving galvanized reinforcing bar». Corrosión 81 , NACE.

3. CORNET, J.; BRESLER, B.: "Critique of testing procedures related to measuring the perfomance of galvanized steel reinforcement in concrete». ASTM STP-713 (1980), p. 160.

4. ARLIGUIE, G.; GRANDET, J. and DUVAL, R.: “Etude du contact zinc-pâte de ciment portland». 7ème Congrés Int. de la Chimie des Ciments. Paris, vol. III, paper VII-22 (1980).

5. MOLINA, A.; BLANCO, M. T.; ANDRADE, C.: “Corrosion rate of three different types of galvanized coatings of steel reinforcements in contact with mortar», 9th. Int. Congress on Metallic Corrosion, vol. 1, Toronto, junio (1984).

6. ANDRADE, C.; MOLINA, A.; HUETE, F.; GONZALEZ, J. A.: «Relation between the alkali content of cements and the corrosion rates of galvanized reinforcements". Corrosion of Reinforcements in Concrete Construction, Ed. Alan Crane, Industrial Chemical Soc. London, June (1983).

7. GAIDIS, J. M.; ROSENBERG, A. M.; SALEH, I.: «Improved test methods for determining corrosion inhibition by calcium nitrite in concrete" ASTM STP-713 (1980), p. 64.

8. ANDRADE, C.; ALONSO, C.; GONZALEZ, J. A.: "Ensayos de laboratorio sobre el efecto inhibidor de los nitritos sobre la corrosión de las armaduras». Materiales de construcción, vol. 34, n.o 195 (1984), p. 41.

9. LUGEZ, J.: «Risques de corrosion des armatures dans les sandwiches lourds béton-polystyrene expansè", Cahiers de CSTB, mayo (1985), p. 33. 